\title{
¿Es posible invertir la forma en que aprendemos y enseñamos? Aderezos para repensar la educación
}

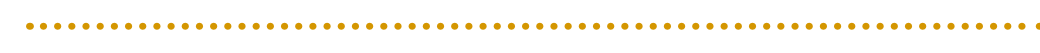

1. Investigadora asistente del Consejo Nacional de Investigaciones Científicas y Técnicas, Universidad Nacional de Río Cuarto, Córdoba, Argentina

2. Investigador principal del Consejo Nacional de Investigaciones Científicas y Técnicas, Universidad Nacional de Río Cuarto, Córdoba, Argentina

\section{Resumen}

El presente ensayo recoge reflexiones sobre experiencias educativas a través de postulados que marcan aspectos clave para repensar los contextos de aprendizaje y enseñanza. Se muestran formas innovadoras y su contraparte al orientar las prácticas educativas dentro del aula. Se presentan argumentos y razones con sustento en las investigaciones de diseño y desarrollos teóricos de la Psicología Educacional para entender y transformar la educación. Consideramos importante rever y reflexionar los roles en educación, la importancia de los contenidos, el papel de los docentes y de los alumnos, para promover la implicación. El trabajo gira en torno a 'aderezos' organizados en cuatro pares contrapuestos, a saber: docentes deliveries versus colaboradores; enseñanza pareja versus diversa; evaluación estándar versus a la carta; aprendizaje invisible versus visible. Cada par delimita aspectos esenciales para pensar la escuela de cara a los retos del nuevo siglo, a partir de una nueva forma de concebir la enseñanza, el aprendizaje y la evaluación. Invitan a salir de la zona de confort a educadores y educandos y los desafía a transitar nuevos caminos más abiertos e inclusivos.
\end{abstract}

Daiana Yamila Rigo' \& Danilo Donolo daianarigo@hotmail.com donolo@gmail.com

Palabras clave: aprendizaje, ambiente educativo, compromiso, Psicología de la Educación, innovación educacional, tendencia educacional.

\section{Abstract \\ Is it possible to reverse the way we learn and teach? Dressings to rethink education}

The present essay offers reflections on educational experiences through postulates that mark key aspects to rethink teaching and learning contexts. We show innovative ways to and its counterpart to guide educational practices in the classroom. We present arguments and reasons to support in research design and theoretical developments of Educational Psychology to understand and transform education. Consider important to review and reflect the roles in education, the importance of content, the role of teachers and students, to promote involvement. The work revolves around 'dressings' organized in 4 opposing pairs, namely: delivery vs. collaborator teaching; couple vs. diverse teaching; 
standard vs. to the letter evaluation; invisible vs. visible learning. Each pair defines essential aspects to think about school facing the challenges of the new century from a new way of thinking about teaching, learning and assessment. Invited out of the comfort zone in which teachers and learners is comfortable in everyday life and challenges them to move new more open and inclusive ways. It shows how the positions and theories in education have their correlates in practice; and that at least part, what we show here. In short, we aim through these postulates, continue to discuss how we can rethink education, the way we learn and teach to configure contexts promising to engage the student?

Key words: Learning, educational environment, engagement, Educational Psychology, educational innovations, educational trends.

\section{CONSIDERACIONES GENERALES... ¿QUÉ IMPLICA INVERTIR LA EDUCACIÓN?}

No es nuevo que muchas veces los alumnos se aburran en la clase, que no presten atención, que se muestren desinteresados hacia los contenidos que la escuela ofrece en su abanico de alternativas a lo largo de los años y sus niveles. Tampoco es novedoso escuchar a los docentes protestar por la desmotivación de los estudiantes, la falta de estudio y esfuerzo. Menos aún es extraño leer y escuchar que la educación necesita un cambio.

En este escrito exponemos argumentos y razones sobre la necesidad de considerar los estudios actuales de la Psicología Educacional para entender y transformar la educación. Consideramos importante rever y repensar los roles en educación, la importancia de los contenidos, el papel de los docentes y de los alumnos. En este marco, presentamos 'aderezos' que nacen de prácticas de investigación llevadas a cabo en contextos educativos formales en interjuego con posturas teóricas actuales, con el objetivo de delimitar aspectos esenciales para pensar la implicación a partir de una nueva forma de concebir la enseñanza, el aprendizaje y la evaluación.

Partimos de considerar fundamental reflexionar sobre cuatro supuestos que conviven entre los muros de las instituciones educativas y que se expanden más allá de ellos, lo anterior con el fin de repensar las ideas y los sentidos de la educación. Supuestos necesarios de derribar y desmitificar para co-construir nuevas perspectivas que permitan que otra educación emerja para el mundo actual.

El primero supuesto está bastante instaurado no sólo entre docentes y alumnos, sino también entre la gente común, es que el aprendizaje ocurre en la cabeza de los sujetos, sin importar mucho el contexto que lo rodea. De ello se deriva que el aprendizaje es un resultado individual, más que grupal.

Una perspectiva, que ayuda a creer en lo contrario resulta de los postulados socioculturales, desde donde se reconoce que los sujetos "funcionan como personas más el entorno porque eso les permite desarrollar mejor sus aptitudes e intereses" (Rinaudo, 2007, p. 13). Es decir, atender a una idea de inteligencia distribuida y saberes compartidos enfatizando que es más fácil aprender cuando contamos con la colaboración de personas y recursos que pueden mejorar nuestra comprensión de las cosas.

Si nos acercamos al concepto de implicación, algo simular se formula desde los modelos contextuales sobre el estudio del compromiso. Una propuesta es la de Lam, Wong, Yang y Lui (2012), la cual remarca la importancia tanto de los rasgos personales, como de los factores contextuales para comprender el compromiso de alumnos y docentes. El cómo ese entorno es percibido por los estudiantes y configurado por los docentes son dos piezas clave junto a las creencias y motivaciones. Tal es así, que $76 \%$ de las variaciones en los niveles de compromiso se deben a las variables que definen al espacio educativo y lo restante a dimensiones asociadas al sujeto que aprende (Shernoff, 2013). 
Asumir los postulados nos permite pensar en la importancia de conformar contextos y recursos educativos para aumentar las posibilidades de experimentar aprendizajes significativos junto a otros. Dejar atrás la idea de trabajo individual y pruebas a solas, implicaría, en parte, revalorizar el valor de atender de manera particular el diseño y la planificación de las actividades académicas, conformando prácticas colectivas para alcanzar un mayor compromiso con el objeto de estudio, que a la vez facilite mejores y más aprendizajes.

Al respecto, Coll (2004) postula la importancia de definir las aulas como comunidades de aprendizaje, donde un grupo de personas con diferentes niveles de experiencia, conocimiento y pericia, aprenden mediante su implicación y participación en actividades auténticas y culturalmente relevantes, y que gracias a la colaboración establecida entre sí y las ayudas que se prestan mutuamente construyen colectivamente el conocimiento.

La segunda creencia, bastante relacionada a la anterior, formula que los docentes son los únicos dueños del saber. Krechevsky y Stork (2000) consideran que la práctica de concebir la enseñanza y la trasmisión de los conocimientos hacia una sola dirección, se relaciona con la creencia de considerar al maestro como consumidor, más que como generador de conocimientos y saberes. Recientemente, por el contrario, se ha puesto mayor énfasis en la construcción colaborativa, en tanto se ha demostrado que los estudiantes no siempre experimentan el contexto de aprendizaje en la manera que fue configurado por los docentes (Könings, Brand-Gruwel, Van Merriënboer, Broers, 2008).

La percepción del contexto educativo está determinada por una multiplicidad de factores, y específicamente la investigación del aula debe optar por una visión integrada, donde el maestro estudie y sea estudiado al mismo tiempo por los alumnos y el currículo (Donolo y Baquero, 2014). Consideramos, sin embargo, que esas discrepancias no son resistentes al cambio, si bien docentes y alumnos poseen su propia perspectiva sobre el proceso de aprendizaje, la participación conjunta en el diseño de la clase y las tareas, puede ser aún mayor, en vista a promover la implicación de ambas partes.

Al respecto, Rinaudo (2014) sostiene que desde comienzos de la década de 1980, una de las grandes críticas al campo de estudios de la Psicología Educacional apuntaba a su desinterés por los problemas reales de aprendizaje. El conocimiento sobre enseñanza y aprendizaje era generado fuera de la clase y ofrecido a través de lecturas, cursos, conferencias o congresos. Asumir lo contrario, implica entrar al aula y documentar las experiencias de aprendizajes que pasan allí dentro, a modo de construir colaborativamente y de manera respetuosa la teoría desde la práctica, modificada y validada por los educadores en el mismo quehacer diario de enseñar y aprender.

Otro elemento del proceso educativo que también es parte de los supuestos que obstaculizan invertir la educación refiere a la evaluación como sinónimo de nota y resultado final. La prueba, el examen o el parcial, dependiendo del ciclo educativo que se esté transitando, suele ser parte de un ritual que se celebra al finalizar un tema, para valorar aquello que los estudiantes fueron capaces de aprender o de retener. Una perspectiva que puede ayudarnos a superar esta concepción se une al concepto de proceso, documentar cómo los estudiantes están comprendiendo lo que aprenden, más que sólo valorar el producto final. Supone pasar de concebir a la evaluación como resultado (sustantivo) y poner el foco en las acciones (verbo) que llevan a cabo conjuntamente alumnos y docentes mientras están desempeñándose en actividades auténticas y contextualizadas.

Por tanto, desde el enfoque no tradicional se pone énfasis en una visión integrada de evaluación e instrucción, como procesos recursivos y no como momentos diferenciados en tiempo y espacio. Pensar que en el parcial, o solo en 60 minutos, alguien puede dar cuenta de su comprensión y aprendizaje es complejo; el pensamiento fácilmente se disuelve al mencionar que la práctica de aprender es constante y a lo largo de toda la vida. Se trata de pasar de una educación basada en la certificación, a una basada en el aprendizaje, que se sustente en las motivaciones de los estudiantes, en responsabilidades a largo 
plazo y el trabajo colaborativo, que permita a la vez medir el progreso en el aprendizaje de los alumnos y planear mejoras en el plan de estudio.

La evaluación de los progresos informa al profesor y a los estudiantes si se necesita más tiempo y cuáles son los procedimientos por seguir. Al respecto, se sostiene que ofrecer un feedback inmediato mientras los alumnos están involucrados en las tareas, colabora significativamente con el aprendizaje, motivando a los estudiantes y generando mejores resultados; aún más productivo si ofrece acceso a los fundamentos y a las explicaciones sobre las sugerencias y correcciones (Brophy, 2002; Garello y Rinaudo, 2012; Rigo, 2016a; Rigo, 2016b).

La última creencia sostiene que la enseñanza y el aprendizaje son actividades más cognitivas, que estéticas, emocionales, éticas y filosóficas. Por mucho tiempo sólo se estudió el componente cognitivo del compromiso, y no fue hasta hace poco que su definición y estudio avanzó hacia una perspectiva más compleja, integrando lo conductual y lo afectivo.

Desde una perspectiva sociocognitiva, como mencionamos anteriormente, se remarca la importancia de entender de una manera más completa los procesos de aprendizaje llevados a cabo por los estudiantes considerando las interacciones que se establecen entre aspectos personales, cognitivos, motivacionales y emocionales, y rasgos particulares del contexto de aprendizaje (Pintrich, 2000). Respecto de las emociones, aunque tienen el potencial de influir en los procesos de enseñanza y de aprendizaje en contextos académicos, su investigación específica en contextos educacionales, emerge tardíamente (Schutz y Pekrun, 2007). En la actualidad, bajo la denominación de emociones de logro, se busca explicar y revalorizar el papel de los componentes afectivos sobre los aprendizajes, pensar al aula como un lugar cargado de emociones para atender a los rasgos que puedan estar contribuyendo a promover el disfrute de los estudiantes por el conocimiento (Schutz y Pekrun, 2007; Pekrun, 2014).

En relación con lo estético y filosófico, Gardner (2006), menciona la importancia de incluir múltiples sistemas simbólicos para ampliar la comprensión de los estudiantes, como forma de indagar los conocimientos propios de una disciplina a partir del lenguaje de otras áreas del saber que contribuyan a propiciar lecturas y significados diversos. Asimismo, agrega el valor de formar personas éticas y socialmente responsables, como pilares para lograr un buen trabajo que tenga significado para sus pares y la sociedad en general (Gardner, 2005).

En este marco, consideramos que lo primero para avanzar hacia una nueva configuración en educación es superar las creencias negativas y consolidar las alternativas positivas y prometedoras. Para conseguirlo, los educadores debemos salir de nuestra zona de confort y generar micro-revoluciones en el aula, es decir, empezar a mirar a la educación desde una óptima distinta, más reflexiva, analítica y autocritica, de manera gradual, paso a paso (Acaso, 2015) y crear mini-contextos educativos que ayuden a plasmar las nuevas ideas y hallazgos en la tarea de enseñar y aprender de alumnos y docentes.

Constantemente, los contextos educativos se renuevan y junto a los avances de las nuevas tecnológicas exigen reflexionar sobre cómo educar a las nuevas generaciones. Consideramos como piezas clave en el desafío la formación de los docentes y la investigación en contextos naturales de enseñanza-aprendizaje, para seguir pensando y rediseñando los entornos educativos.

Pero ¿por qué insistimos en invertir la educación?, porque nos parece un concepto potente para incentivar acciones que generen las micro-revoluciones en el aula. La palabra invertir dice la Real Academia Española de la Lengua puede tener al menos tres acepciones, en principio implicaría cambiar, sustituyendo por sus contrarios, la posición o el sentido de las cosas; en segundo lugar, supone emplear, gastar, colocar un caudal; por último, podría referir a emplear u ocupar el tiempo.

Así, invertir la educación supone, en primer lugar cambiar, romper, sustituir cada supuestos por sus contrarios y en segundo lugar, supone disponer de tiempo para pensar y para realizar nuevas búsquedas 
y lecturas, implica transformar las aulas, las concepciones de los alumnos y los docentes sobre enseñanza, aprendizaje y evaluación, apostando a la formación de los docentes para lograr tal inversión. Se trata de dar vuelta la página y de eso nos ocuparemos en "la esencia de los aderezos" que funcionan como esos contrarios necesarios para trasformar los contextos educativos en pro de alumnos y docentes más comprometidos.

En tal sentido, presentamos a continuación cuatro aderezos, que describimos en detalle, pretendiendo contribuir, aunque sujetos a nuevas redefiniciones cuando la práctica y la realidad educativa así lo requieran, a promover el compromiso de docentes y alumnos hacia el aprendizaje y la educación.

\section{LA ESENCIA DE LOS ADEREZOS}

Si invertir decíamos que es sustituir por contrarios, aquí presentamos cuatro aderezos que a modo de pares contrapuestos muestran prácticas que se deberían sustituir por experiencias que valga la pena poner en primer plano en pos de una educación orientada a promover la implicación (A los efectos didácticos usamos el recurso de los extremos contrapuestos para mostrar mejor las diferencias y las potencialidades. Tal vez en posiciones menos extremas nos sintamos mejor con las teorías y con incluir algunos cambios progresivos en nuestra práctica educacional.

\section{Docentes deliveries versus colaboradores}

Estamos acostumbrados a pensar que los docentes entregan conocimiento, al considerar que el maestro es dueño del saber y que nadie más que él tiene ese poder. La escuela funciona en modo delivery, algo así como llamar y pedir una pizza, confiados en que entregarán la que hemos solicitado, tal cual la imaginamos y en el tiempo estipulado. Pensar la docencia como una simple oferta de saberes, es considerar a los estudiantes como sujetos pasivos en la apropiación de los contenidos, es suponer que la trasmisión es receptada tal cual fue emitida. En realidad, poco se recuerda el contenido enseñado en el aula bajo el modelo de trasmisión, por el contrario, el aprendizaje ligado a lo experiencial, es aquel que tiene mayores oportunidades de perdurar en los recuerdos.

En el modelo tradicional de enseñar y aprender, los docentes recitan de sus borradores y los alumnos toman apuntes, o como actualmente se acostumbra, realizan una copia en fotografía de las notas de los profesores sobre el pizarrón, y asumimos en definitiva que todo acontece sin filtros, ni modificaciones; realmente ¿es así?, ¿qué pasa con la pluralidad de interpretaciones?, ¿de perspectivas?, ¿de significados? No será mejor que empecemos a pensar que los alumnos realizan una lectura particular de los conceptos e ideas que toman un sentido particular en relación con sus vivencias, sentimientos, motivaciones, intereses y percepciones.

En este marco, cobra relevancia la idea de un diseño colaborativo del currículo entre docentes y alumnos, en contraposición al docente como proveedor de conceptos y contenidos. Harris (2010) señala que los docentes que apuestan a crear de manera colaborativa los contextos de enseñanza y aprendizaje, son aquellos que poseen una concepción sobre el compromiso y su forma de cultivarlo, lo cual se halla en la línea de los planteamientos que aquí venimos sosteniendo, una implicación que necesariamente se promueve si se considera al otro como sujeto activo de aprendizaje, que construye y significa los aprendizajes en función de sus experiencias previas, que se motiva y participa en tareas afines a sus intereses.

De igual manera, sería interesante pensar el lugar de los docentes con la gran influencia de las nuevas tecnologías en educación; rodeados de Internet, con un sinnúmero de sitios virtuales que llevan como prefijo wiki (del hawaiano wiki, 'rápido'), como la famosa Wikipedia. En esos entornos los usuarios pueden 
crear, editar, borrar o modificar el contenido del sitio, de una forma interactiva, fácil y rápida; haciendo de la wiki una herramienta efectiva para la escritura colaborativa. Una manera de construir el saber junto a otros. Una colaboración que se monta en esa 'nube' (Cloud computing) que atrapa y acapara el conocimiento que viaja de sujeto en sujeto, de ciudad en ciudad, de país en país, de continente en continente. Un saber que está en permanente modificación y actualización, en busca de mejores respuestas. Un saber que es narrado por sujetos, que supera ideas recibidas por otros, a partir de la confrontación y el contraste de observaciones y relatos sobre un mismo objeto de conocimiento.

Entonces, por qué presentar al conocimiento de manera acabada, como si no existiera un proceso de construcción continuo. Consideramos que el punto central para atender a prácticas educativas de colaboración se acerca a la nueva concepción de retroalimentación, alejada de la señal o información que se transmite de un emisor a un receptor, y que toma proximidad con la idea de abrir un diálogo que entrelace a profesores y alumnos en torno de etapas particulares en el desarrollo de tareas de aprendizaje (Rinaudo, 2014). Pensar la educación más allá de lo prefijado, supone a docentes que se alejen de la comodidad de la respuesta hacia la incomodidad de la pregunta, maestros que escuchen y se dejen interpelar por un otro, con conocimientos y expectativas propias y distintas (Caminos de $t$

iza, 2016a; 2016b; 2016c). En esta línea, las herramientas virtuales pueden ayudar a mantener el diálogo recursivo entre docentes, algunas y también familiares, como ClassDojo (nd) que facilita crear una comunidad de clase, que amplía el modo en que los maestros pueden dar a sus estudiantes retroalimentación de su comportamiento tanto cognitivo-afectivo como conductual, gestionar sus clases y ofrecer a los padres indicadores sobre el progreso de sus hijos en la escuela. Aspectos que, investigaciones actuales vienen remarcando como positivos para pensar la implicación escolar (Santos, Godás y Modelo, 2016).

La propuesta es generar un ambiente de aprendizaje que simule el aula como una gran Wiki, escuchar los saberes y las perspectivas de cada niño, permitir que el pensamiento se externalice, que cada cual narre sus experiencias, emociones e interés, y construir un relato que comparta la multiplicidad de voces, que se redefina en función de nuevas lecturas, búsquedas y explicaciones. Bruner (1997) propone en este desafío, no sólo construir comunidades de aprendizaje, sino rescatar la actividad cognitiva del estado implícito, haciéndola más pública, negociable y solidaria.

Al respecto, Morán Oviedo (2012), reflexionando sobre el rol de los docentes, entiende que la tarea de transmitir un conocimiento en la perspectiva "de quien sabe a quién no sabe", puede resultar sencilla, consabida y hasta cómoda. Por el contrario, involucrarse teórica y metodológicamente en un proceso de enseñanza-aprendizaje, donde el vínculo profesores-alumnos se conciba como fenómeno complejo, supone dejar de considerar a los educandos como objetos de enseñanza y pasar a ser sujetos de aprendizaje.

Se trata de apostar a una escuela que se des-focalice de los desempeños individuales y apueste por el saber socialmente compartido, que se aparte de la memorización hacia la construcción conjunta de nuevos saberes. De ser docentes que enseñan a la vez que aprenden, y ser alumnos que aprenden a la vez que enseñan. Algunas experiencias, muestran este camino, tal como Getting Smart (nd), una comunidad sobre innovación docente que ofrece un espacio virtual de colaboración para que maestros de todo el mundo compartan sus experiencias de cambio, sus pasiones, investigaciones y planes de actividad a modo de generar una instancia de intercambio enriquecedora, una modalidad de trabajo en conjunto para crear experiencias educativas de gran alcance para los estudiantes de distintos lugares.

\section{Enseñanza pareja versus diversa}

Nos preguntas por qué en vez de leer todos los niños del mismo manual, no dejamos que ellos elijan qué y cuándo aprender. Por qué no ofrecemos en el aula espacios para preguntar y recursos que inviten a dar 
respuestas momentáneas, no definitivas, que podrán tener nuevas argumentaciones en experiencias posteriores o conversaciones futuras. Hoy sabemos que no todos aprendemos igual, por suerte, hay diversidad en la forma de hacerlo y también intereses que muestra que las preocupaciones van en sentidos múltiples al igual que las motivaciones para aprender. En un ejemplo concreto, pensar que los motivos para aprender de un universitario son los mismos que las de un adulto mayor, nos haría caer en una falsa representación sobre las razones que mueve a uno u otro grupo a estar un contexto de aprendizaje.

Swartz, Costa, Beyer, Reagan y Kallich (2008) y Perkins (2008) postulan la importancia de ofrecer un escenario que estimule un aprendizaje basado en el pensamiento, donde el conocimiento cobre vida, se active para realizar conexiones y predicciones, para crear productos y conseguir resultados creativos. Es decir, proponen como meta alcanzar un aprendizaje pleno, que responda al ¿para qué se aprende esto? o ¿cuál es el valor de utilidad de los contenidos escolares?, se busca así nuevas formas de propiciar la transferencia a situaciones lejanas, donde el conocimiento no queda en estado inerte en un cuaderno o en una hoja de evaluación, sino que se expande más allá de las paredes del aula. Una práctica educativa que ayuda, en el sentido expresado, son las ferias de ciencias escolares que motivan a generar ese puente entre lo que se aprende y problemáticas de la comunidad donde se inserta la escuela, investigando situaciones que afectan de manera directa a docentes y estudiantes, una forma de volcar la aprendido en el aula para solucionar y generar conocimientos con sentido cultural.

Un enseñanza despareja la vinculamos también con prácticas educativas originales e inesperadas, que movilicen desde otro lugar las estructuras cognitivas de los alumnos y docentes. Mostrar otras cosas, hacer algo distinto en el aula o fueras de ella, para encontrar otro sabor a lo aprendido. Desde la Psicología Educacional, se postula que las tareas académicas que se definen como originales o novedosas muestran un impacto importante en los aprendizajes de los estudiantes, tanto a nivel cognitivo, como afectivo y conductual (Ames, 1992; Rigo y Donolo, 2014).

Acaso (2015) postula la importancia del extrañamiento como herramienta pedagógica. Remarca que la sorpresa, el asombro y lo inesperado son factores señalados desde la neuroeducación como necesarios para que el aprendizaje suceda. Explica que el extrañamiento es un mecanismo que consiste en provocar un efecto de sorpresa en los destinatarios de la acción quienes desarrollan una sensación de 'efervescencia intelectual', que prepara el mejor escenario para que ocurra el aprendizaje.

Asimismo, Ballarini (2015), desde la neurociencia, menciona que las experiencias novedosas no relacionadas con lo estrictamente académico o por lo menos con el contenido por enseñar, produce un efecto positivo en la memoria de los estudiantes. Es decir, si leemos un cuento a los estudiantes antes de presentar la teoría de la relatividad, el entorno inesperado prepara la antesala para generar un mayor interés y por tanto una mayor retención de lo enseñando. Precisamente, los resultados de un experimento conducido por el mismo autor, muestran que eso realmente sucede al comparar dos grupos. Uno de los experimentos consistió en leer a los alumnos cuentos de Ema Wolf, una hora antes de dictar una clase de ciencia a algunos alumnos de cuarto grado. Al día siguiente, se les tomó un examen relacionado a la temática estudiada, se concluyó que los estudiantes que presenciaron el 'evento novedoso' obtuvieron mejores resultados que quienes no.

Las implicancias educativas de estas prácticas son importantes para repensar la labor decente con base en los intereses y curiosidades de los estudiantes. En este sentido, en la formulación de las tareas académicas se deben tener en cuenta dos componentes centrales: que sean desafiantes, a nivel cognitivo, a la vez de interesantes, para asegurar un aprendizaje significativo y por tanto a largo plazo. Afirmamos así: aquello que impacta y sorprende queda para toda la vida en nuestra memoria.

Ciertamente, involucrar a los estudiantes en experiencias novedosas, tiene un impacto en el almacenamiento a largo plazo (Ballarini, 2015), lo cual es compartido por los estudiantes, quienes en un estudio relacionado con incluir el arte y lo estético en una clase de ciencias sociales en el nivel primario de 
educación, señalan haberse comprometido más con la aprendido, pero también recordar más lo estudiado (Rigo, 2014). Una experiencia similar, pero con estudiantes de nivel universitario, muestra cómo el generar prácticas inesperadas también contribuye a ampliar las zonas de aprendizaje de los estudiantes (Rigo y Damilano, 2015).

Por último, en una enseñanza despareja, no consideramos menos importante repensar los espacios donde se llevan a cabo las práctica educativas, aunque pareciera hasta ingenuo preguntarnos ¿por qué aprendemos dentro del aula?, responderla conlleva a pensar en otros escenarios posibles e igualmente interesantes, pero ¿qué es el aula?, si nos remitimos a las experiencias personales son lugares con bancos alineados en un mismo sentido, y un escritorio que los enfrenta en dirección contraria, junto a un pizarrón donde se deja registro de lo que acontece en ese espacio; habitada con uniformes más o menos idénticos, sentados cada cual en su lugar y un adulto que, deambulando por los estrechos espacios entre banco y banco, va recitando la clase. Aunque tradicionalmente esta imagen está en el recuerdo de muchos de los que hemos transito la educación formal, ¿se podrá aprender en otro espacio? en una plaza, un museo, a distancia. Efectivamente el aprendizaje tiene lugar en varios entornos, incluso en la 'nube', en modalidad a distancia, en clases invertidas.

Estudios actuales indican que el modelo de la educación invertida, que busca integrar nuevas tecnologías en la enseñanza de contenidos utilizando activamente materiales audiovisuales (Hutchings y Quinney, 2015; Jamaludin y Osman, 2014), contribuye de manera directa sobre la implicación de los estudiantes, en tanto responde a las nuevas características que definen a los estudiantes de hoy, ligados a las nuevas tecnológicas y, en palabras de Nieto (2012), como un alumno que "escucha más que ve, y ve más que lee" y responde a lo que Wales (2015) menciona como el nuevo reto educativo "el nuevo analfabetismo no es no saber cosas, es no saber usar la información".

\section{Evaluación estándar versus a la carta}

Para ir a comer, uno podría optar por un restaurante que ofrece un menú fijo cada día o bien optar a uno que ofrezca diversas opciones a la carta. Algo similar se percibe en educación. Una primera apreciación, se podría realizar respecto a las materias y su organización, pareciera que existe un orden más o menos establecido e inmóvil en relación con las materias en una semana escolar: una hora de música, otra para educación tecnología, otro más para educación física, un poco más, alrededor de tres horas, para ciencias sociales y naturales y el resto, todas esas horas que faltan para completar las tareas que se desarrollan todos los días, de 8 a 12, son destinadas a lengua y matemática. Una segunda lectura, ya sobre la evaluación estrictamente, nos remite a un tiempo y un espacio homogéneo de responder preguntas, un marco establecido para certificar qué se sabe y qué se aprendió. Pareciera que la educación obedece a formatos más o mensos fijos e iguales para todos, contrario de la diversidad que nos caracteriza como sujetos, trata de simplificar en alternativas acotadas, estilos y maneras de hacer, decir y pensar muy distintos.

Desequilibrar la evaluación, también nos lleva a pensar en los tipos de preguntas que más frecuentan los pizarrones de las aulas, en primer lugar advertimos que predomina aquellas preguntas que etiquetamos como fácticas, por solicitar información concreta, fácil de encontrar en los libros, que implican poco desafío cognitivo (Furman, 2015). En segundo lugar, la formulación de preguntas en el aula de clase ha sido tradicionalmente una tarea casi exclusiva de los docentes, ya sea como parte de las tareas académicas, 0 bien de la evaluación; interrogantes que muchas veces coinciden en una y otra instancia. De tal manera, que los estudiantes cuentan con pocas ocasiones de formular preguntas, indagar más allá del libro o de manual de ciencias ${ }^{21}$. 
Ahora bien, cómo reorientamos las prácticas, cuáles alternativas se presentan como posibilitadoras de una evaluación a la carta. En principio, fusionar instrucción y evaluación puede ser un camino que facilite un aprendizaje basado en situaciones problemáticas reales, es decir, propuestas que los alumnos puedan resolver desde sus conocimientos previos, pero que además se les presente el desafío de buscar nueva información para completar la solución del problema formulado, que a la vez está asociado a circunstancias próximas de su vida fuera de la escuela. En la misma no se evalúa contenidos ni datos, sino más bien la comprensión de poner en juego los conceptos centrales del currículo para analizar una situación cotidiana (Rigo y Donolo, 2016; 2017).

Hablar de la evaluación como experiencias o situaciones por resolver, busca rescatar el interés de los alumnos, su participación e implicación, dejar de contemplar para vivenciar el aprendizaje, crear un puente entre lo que ocurre en el aula y el exterior. Ofrecer la oportunidad de elegir qué aprender y en qué momento, estimulando la toma de decisiones y la autonomía; se abre un lugar para gestionar los propios procesos cognitivos puestos en marcha durante la tarea de aprendizaje, habilitando un escenario de evaluación que, como proceso de andamiaje, regula la construcción del conocimiento.

En estas experiencias, el saber se vuelve provisorio, sujeto a cambios y a nuevas preguntas que conducen a nuevas búsquedas. Encamina a pasar de una educación basada en la evaluación, a una basada en el aprendizaje, en tanto, los conocimientos, comprensiones y saberes de los estudiantes ya no son representados sólo a partir de un número (Camussi, 2015). Por eso, las propuestas actuales resaltan la importancia de evaluar el proceso, y no solo el producto final.

En este marco, herramientas como las rúbricas, posibilitan compartir y exponer con claridad los criterios de evaluación, que guían y moldean el comportamiento de los estudiantes, en tanto conocen qué se espera de cada experiencia de aprendizaje. La incorporación de rúbricas como herramienta de evaluación facilita y motiva la participación y una mayor responsabilidad de los estudiantes en el proceso evaluador, generando instancias que facilitan la autorregulación y la retroalimentación; que contribuyen a monitorear y gestionar el propio aprendizaje, y favorece el conocimiento de los objetivos de una tarea y el progreso hacia su logro.

Al respecto, los estudios muestran que los estudiantes destacan el uso de los criterios para planificar, monitorear y reflexionar a lo largo del proceso de resolver la tarea, como un recurso que ayuda a comprender los objetivos de aprendizaje, así como de los criterios de desempeño que guían las producciones (Rigo, 2016b). Es decir, la rúbrica funciona a modo de mapa o receta para orientar la toma de decisiones o cambios en las estrategias, promoviendo feedbacks que llevan a adoptar una perceptiva crítica sobre la propia producción. Retroalimentaciones que, en torno a los criterios, ayudan a comprender las cualidades que el trabajo debe poseer, habilitando un espacio para revisar y mejorar la tarea o el resultado final, ofreciendo a los alumnos la posibilidad de valorar sus producciones contando con información valiosa acerca de las metas de aprendizaje y los niveles de calidad por alcanzar (Andrade y Du, 2005; Andrade y Valtcheva; 2009; Gallego y Raposo, 2014). Lo anterior, atiende de manera particular a promover el compromiso cognitivo, estimulando la autorregulación.

Podríamos decir que, desde esta nueva perspectiva de evaluación, el aprendizaje se convierte en un bufé a la carta, donde los estudiantes puede elegir qué investigar, en qué tiempo y con qué recursos, el plus de este tipo de prácticas está en la promoción de la capacidad de auto gestionar el propio aprendizaje, la autonomía que ganan los alumnos en la toma de decisiones, así como el mayor compromiso y responsabilidad con respecto de sus estudios. En esta inversión, la reorganización del currículo no por materia, sino por interés, abre una nueva posibilidad de aprendizajes situados e interconectados y más autónomos. 


\section{Aprendizaje invisible versus visible}

Empezamos este último aderezo con la siguiente pregunta, ¿qué tipo de pensamiento se privilegia y se cultiva en el aula? En la mayoría de los contextos formales, los docentes solicitan a los estudiantes que completen tareas y actividades. Nada de malo muestran estas prácticas, dado que bien diseñadas y orientadas pueden ayudar a promover una mejor comprensión de los temas en cuestión, sólo que a veces se dejan al azar aquellas tutorías que podrían conducir a un verdadero entendimiento, cayendo en la reproducción de habilidades y conceptos. Acaso (2015), utiliza la expresión 'estudiantes bulímicos' para aludir a una práctica bastante común entre los alumnos, que implica básicamente memorizar el material de estudio para volcarlo al papel de evaluación, sin dejar rastros en la memoria tras el eventual examen.

Así, hacer visible el pensamiento se relaciona con las posibilidades de poder acceder a él tantas veces sea necesario y útil en otros contextos, más alejados del aula y la escuela. Se asociada a un saber que puede ser apropiado y resignificado por el sujeto que aprende, un conocimiento distinto al inerte, que perdure más allá de las evaluaciones y de las paredes del aula. Un saber al cual se puede recurrir una y otra vez, en entornos de usos diversos. Al respecto, las tareas académicas que se caracterizan por ser auténticas, con valor de utilidad y contextualizadas conducen a tal objetivo (Rigo, 2017; Rigo y Donolo, 2014).

En esta línea, también debemos destacar aquellas prácticas de enseñanza algo provocadoras para incitar a reflexionar. En una charla de Café Científico, Donolo (2016), profesor consulto de la Universidad Nacional de Río Cuarto, enseñó con sutileza sobre el valor de la duda y a la vez ofreció una nueva herramienta para orientar el pensamiento. Su postulado es perspicaz, no creerse todo lo que se dice, siempre hay mejores respuestas. Si nos animamos a dudar un poco de aquello -Internet- o aquellos -docentes- que acostumbramos a creer que nos dicen la verdad, y alentamos la búsqueda de nuevos caminos para comprobarlo, puede que tengamos una forma de incentivar la adquisición de conocimientos para asumir con argumentos y matices diversos la información recibida, y de discutir junto a otros sobre el valor y lo fiable de la información que se dispone en una pantalla, en un pizarrón, en una conferencia o en la televisión.

Es otra forma de hacer visible lo invisible, de implicar cognitivamente al que aprende y de ampliar las posibilidades de saber y encontrar nuevos conocimientos y matices. Al respecto, Ritchhart, Church y Morrison (2014) plantean en la misma línea que, en lugar de buscar o aceptar las respuestas fáciles, es interesante avanzar un poco más allá, e identificar la complejidad de los eventos, las historias y las ideas que tenemos frente a nosotros. Lo que implicaría abandonar el confort de la complacencia y aprender a sospechar, lo cual no supone mentir, sino aceptar que no siempre todo es verdad (Acaso, 2015).

Por tanto, formular en educación este tipo de propuesta supone atender de manera particular a preguntas que incentiven la externalización del pensamiento, al respecto Ritchhart, Church y Morrison (2014) postulan cuatro categorías, que permiten para ir más allá de las interrogantes que sólo ponen a prueba la memoria de los estudiantes y que no los involucra con las ideas, sino que sólo guían la revisión del contenido. Las preguntas auténticas, es decir, preguntas que no poseen necesariamente una respuesta o bien para las cuales no hay una sola respuesta posible. Las preguntas generativas, que ayudan a promover la indagación, el descubrimiento y la exploración continua de todo lo que pasa en el aula. Las preguntas constructivas, que animan a conectar ideas, hacer interpretaciones, enfocarse en las grandes ideas y conceptos centrales. Así como las preguntas facilitadoras, que ayudan a los docentes a clarificar el pensamiento de los estudiantes, a modo de promover el pensamiento de los alumnos y su comprensión. Solicitar elaboraciones, justificaciones y argumentaciones que motiven discusiones dentro de la clase, escuchando diversas perspectivas.

Asimismo, hacer visible el pensamiento también supone reflexionar sobre las ideas y desarrollar argumentos propios y genuinos; hacer consciente en el proceso de aprendizaje las estrategias y los procesos 
cognitivos y afectivos que se ponen en marcha cuando se está aprendiendo y se lleva a cabo una tarea académica, es decir, volverse más metacognitivos y autorregulados. Un tema ampliamente estudiado, pero con menor influencia en las prácticas educativas.

La idea es generar un escenario educativo que apoye la autonomía, pero a la vez ofrezca una buena estructura, es decir, que considere la perspectiva de los estudiantes, identifique sus necesidades, intereses y preferencias, que provea de desafíos óptimos, destacando los objetivos de aprendizaje significativo; y simultáneamente comunicar a los estudiantes las expectativas sobre una tarea, ofrecer orientaciones y retroalimentaciones constructivas (Hospel y Galand, 2016; Jang, Reeve y Deci, 2010).

\section{REFLEXIONES FINALES}

Los preguntas en educación nunca terminan, si bien hemos intentado dar respuesta a ¿cómo podemos repensar la educación para promover el compromiso?, nuevas ideas y perspectivas en educación siempre son necesarias para llevar a cabo innovaciones pedagógicas, nada se da por sentado, ni perdura. Los contextos educativos se renuevan y junto a los avances de las nuevas tecnológicas nos exigen seguir pensado cómo educar a las nuevas generaciones, sin caer en trampas o hacer más de lo mismo con título distinto. Consideramos que la formación de los docentes y de los profesores, es una pieza clave para seguir pensando los aderezos presentados, sólo la práctica permitirá validar y redefinir los postulados presentados, ofreciendo nuevos condimentos para rediseñar los entornos educativos.

Las ideas delineadas en el trabajo muestran que la base de la educación son buenas ideas surgidas de buenas prácticas, necesitamos hacer, probar e innovar para crear otras formas de enseñar y aprender que respondan a los intereses y gustos de los alumnos. Necesitamos estar atentos a los retos que las sociedades marcan para la educación, no puede ser que tengamos una educación del siglo XIX, docentes del siglo XX y estudiantes del siglo XXI.

Creer que el conocimiento se halla en la cabeza más que distribuido, que los docentes son sólo consumidor de teorías, que la evaluación es una instancia final o que el aprendizaje es solo cognitivo y no afectivo, frena el avanzar en el sentido que los cuatro aderezos nos invitan.

Apostamos a crear nuevas zonas de aprendizaje y nuevos escenarios educativos, sabiendo que cada nuevo ingrediente genera sabores y gustos alternativos para la educación, a ser novedosos y originales en las formulaciones dentro del salón de clase, sin descuidar la fundamentación teórica de las propuestas implementadas.

Por último, serán bienvenidas las sugerencias de quienes se animen a probar el coctel preparado para un quehacer distinto en educación, a modo de ir generando comunidades de aprendizaje que nos permitan ir revisando constantemente si los aderezos aún tienen sentido o debemos revisar de nuevo la práctica junto a la teoría para emprender originales senderos educativos y nuevas prácticas que apunten a crear entornos colaborativos, donde instrucción y evaluación se fusionen posibilitando el pensamiento visible a través de una enseñanza y un aprendizaje variado e ilimitado en formas, espacios, recursos y motivaciones, en pos de promover el compromiso de los estudiantes.

\section{REFERENCIAS}

Acaso, M. (2015). Reduvolution. Hacer la revolución en la educación. Buenos Aires: Paidós Contextos.

Ames, C. (1992). "Classroom: Goals, structures, and student motivation". Journal of Educational Psychology, 84 (3), 261-271. 
Andrade, H. \& Du, Y. (2005). Student perspectives on rubric-referenced assessment. Practical Assessment, Research \& Evaluation, 10(3), 1-11.

Andrade, H. \& Valtcheva, A. (2009). Promoting learning and achievement through self-assessment. Theory Into Practice, 48, 12-19.

Ballarini, F. (2015). REC. Por qué recordamos lo que recordamos y olvidamos lo que olvidamos. Buenos Aires: Sudamericana.

Brophy, J. (2002). Teaching. UNESCO. International Academy of Education (IAE). Recuperado de: http:// www.ibe.unesco.org/fileadmin/user_upload/archive/Publications/educationalpracticesseriespdf/ prac01e.pdf

Bruner, J. (1997). Educación puerta a la cultura. Madrid: Alianza.

Caminos de tiza (TV Pública Argentina). (2016a). El valor de las preguntas (1 de 4). Recuperado de https:// www.youtube.com/watch? $\mathrm{v}=\mathrm{f} 3 \mathrm{bcs} N \mathrm{NUEX}$ s

Caminos de tiza (TV Pública Argentina). (2016b). El valor de las preguntas (2 de 4). Recuperado de https://www.youtube.com/watch?v=gD_uy6XfbL4

Caminos de tiza (TV Pública Argentina). (2016c). El valor de las preguntas (2 de 4). Recuperado de https:// www.youtube.com/watch?v=kunD7Z3wB0k

Camussi, S. (2015). No soy un siete. Conferencia TEDxRíodelaPlataED. Recuperado de http://www.tedxriodelaplata.org/videos/no-soy-un-7

ClassDojo (nd). Recuperado de https://www.classdojo.com/es-es/?redirect=true

Coll, C. (2004a). Las comunidades de aprendizaje: Nuevos horizontes para la investigación y la intervención en psicología de la educación", IV Congreso Internacional de Psicología y Educación. Almería, España. Consultado 23 de julio de 2009 en http://www.psyed.edu.es/prodGrintie/conf/ CC_Almeria_04.pdf

Donolo, D. (2016). El mundo en un museo jno es lo mismo sin ti! Ciclo 2016 Café Científico. Charla abierta de Divulgación Científica, Letras con Café, Vinculación Tecnológica de la Secretaría de Extensión y Desarrollo de la Universidad nacional de Río Cuarto. 12 de mayo, Río Cuarto, Cba. Argentina. Conferencia.

Donolo, D. \& Baquero, R. (2014). Prólogos. En Paoloni, P., Rinaudo, C. \& González, A. (Comp.), Cuestiones en Psicología Educacional. Perspectivas teóricas, metodológicas y estudios de campo (pp.1132). La Laguna: Sociedad Latina de Comunicación Social. Recuperado de http://issuu.com/ revistalatinadecomunicacion/docs/cde01

Furman, M. (2015). Preguntas para pensar. Conferencia TEDxRíodelaPlataED. Recuperado de http:// www.tedxriodelaplata.org/videos/preguntas-para-pensar

Gallego, M. J. y Raposo, M. (2014). Compromiso del estudiante y percepción del proceso evaluador basado en rúbricas. Revista de Docencia Universitaria, 12 (1), 197-215.

Gardner, H. (2005). Las cinco mentes del futuro. Barcelona: Paidós Ibérica.

Gardner, H. (2006). Multiple Intelligences. New Horizons. New York: Basic Book.

Garello, M. \& Rinaudo, M. (2012). Características de las tareas académicas que favorecen aprendizaje autorregulado y cognición distribuida en estudiantes universitarios. RedU Revista de Docencia Universitaria, 10, 415-440.

GettingSmart (nd). Recuperado de http://gettingsmart.com/publication/getting-smart-teacherscollaborative-curriculum-designers/

Harris, L. (2010). Delivering, Modifying or Collaborating? Examining Three Teacher Conceptions of How to Facilitate Student Engagement. Teachers and Teaching, 16 (1), 131-151. 
Hospel, V. \& Galand, B. (2016). Are both classroom autonomy support and structure equally important for students' engagement? A multilevel analysis. Learning and Instruction, 41, 1-10. Recuperado de: http://dx.doi.org/10.1016/j.learninstruc.2015.09.001

Hutchings, M. \& Quinney, A. (2015). The Flipped Classroom Disruptive Pedagogies Enabling Technologies and Wicked Problems Responding to the Bomb in the Basement. The Electronic Journal of e-Learning, 13(2), 106-119.

Jamaludin, R. \& Osman, S. (2014) The use of a flipped classroom to enhance engagement and promote active learning. Journal of Education and Practice, 5(2), 124-131.

Jang, H., Reeve, J. \& Deci, E. (2010). Engaging Students in Learning Activities: It Is Not Autonomy Support or Structure but Autonomy Support and Structure. Journal of Educational Psychology, 102 (3), 588600. Recuperado de: DOI: 10.1037/a0019682

Könings, K. D., Brand-Gruwel, S., Van Merriënboer, J. J. G., \& Broers, N. (2008). Does a new learning environment come up to students' expectations? A longitudinal study. Journal of Educational Psychology, 100, 535-548.

Krechevsky, M. \& Stork, J. (2000). Challenging Educational Assumptions: lessons from Italian-American collaboration. Cambridge Journal of Education, 30 (1), 57-74

Lam, S-F., Wong, B., Yang, H. \& Liu, Y. (2012). Understanding Student Engagement with a contextual Model. En Christenson, S., Reschly, A., Wylie, C. (Edits.) Handbook Research on Student Engagement (pp.403-420). Minneapolis, MN: Springer.

Morán Oviedo, P. (2012). La evaluación cualitativa en los procesos y prácticas del trabajo en el aula. D.F. México: Universidad Nacional Autónoma de México -Instituto de Investigaciones sobre la Universidad y la Educación. Recuperado de: http://www.acuedi.org/ddata/11345.pdf

Nieto, A. (2012). Los estudiantes y las nuevas tecnologías. Reportaje Recuperado de: http://revistareplicante.com/los-estudiantes-y-las-nuevas-tecnologias/

Pekrun, R. (2014). Emotions and Learning. UNESCO. International Academy of Education (IAE). Recuperado de: http://www.ibe.unesco.org/fileadmin/user_upload/Publications/Educational_Practices/ EdPractices_24eng.pdf

Perkins, D. (2008). Prólogo. La música del conocimiento. En R, Swartz, A., Costa, B., Bayer, R. Reagan, y. B. Kallick (2008). El aprendizaje basado en el pensamiento (pp.7-9). Madrid: Ediciones SM.

Pintrich, P. (2000). The role of goal orientation in self-regulated learning. En M. Boekaerts, P. Pintrich y M. Zeidner (eds.), Handbook of self-regulation (pp: 451-502). San Diego: Academic Press

Rigo D. \& Donolo, D. (2016). La evaluación...más de lo mismo. Desafiando formatos y modalidades sin libreto. Panorama. Revista de Ciencias Sociales, 10 (19): 5-23.

Rigo D. \& Donolo, D. (2017). El valor de utilidad de los contenidos escolares. Percepciones de los estudiantes de nivel primario. Psicodebate, 17 (1): 35-50.

Rigo, D. (2014). Aprender y enseñar a través de imágenes. Desafío educativo. ASRI: Arte y Sociedad. Revista de Investigación, 6: 1-9. Revista Electrónica. Recuperado de: http://dialnet.unirioja.es/servlet/ revista?codigo $=16430$

Rigo, D. (2016a). Planificar, monitorear y evaluar el proceso de aprendizaje: ¿Cómo lo hacen estudiantes de nivel primario de educación? Profesorado. Revista de Curriculum y Formación del Profesorado, 20 (3): 527-548.

Rigo, D. (2016b). Autorregulación y rúbricas como herramienta de evaluación. Experiencia desarrollada en educación primaria. Revista Escuela Abierta, 19: 65-79.

Rigo, D. (2017). Docentes, tareas y alumnos en la definición del compromiso. Investigando el aula de nivel primario de educación. Educação em Revista, 33: 1-24. 
Rigo, D. \& Damilano, G. (2015). Lo inesperado habitando la clase de estadística. Libro de resumen extendido $1^{\circ}$ Congreso Argentino de Estadística CAE (pp. 315-320). CABA.

Rigo, D. \& Donolo, D. (2014). Entre pupitres y pizarrones. Retos en educación primaria: el aprendizaje con compromiso. Educatio Siglo XXI, 32 (2), 59-80.

Rinaudo, C. (2007). 'Caminos de tiza', educación, creatividad y futuro. Coldecón de Cuadernillos de actualización para pensar la Enseñanza Universitaria, 2 (3). Universidad Nacional de Río Cuarto, Sec. Académica, Área de Vinculación. Recuperado de: https://www.unrc.edu.ar/unrc/academica/pdf/ cuadernillo13.pdf

Rinaudo, M. (2014). Estudios sobre los contextos de aprendizaje: arenas y fronteras. En Paoloni, P., Rinaudo, M. \& A. González. Cuestiones en Psicología Educacional. Perspectivas teóricas y metodológicas orientadas a la mejora de la práctica educativa (pp.163-206). La Laguna: Sociedad Latina de Comunicación Social. Recuperado de: http://www.cuadernosartesanos.org/educacion.html

Ritchart, R., Church, M. \& Morrison, K. (2014). Hacer visible el pensamiento. Cómo promover el compromiso, la comprensión y la autonomía de los estudiantes. Buenos Aires: Paidós.

Santos, M., Godás, A. \& Modelo, M. (2016). ¿Puede la implicación de los padres mejorar el estudio de sus hijos en la escuela? La evidencia de un programa pedagógico. Estudios sobre Educación, 30: 9-30. Recuperado de: DOI: 10.15581/004.30.9-30

Schutz P. \& Pekrun R. (2007). Emoción en educación. San Diego. Academic press.

Shernoff, D. 2013. Optimal learning. Environment to promote student engagement. New York: Springer.

Swartz, R, Costa, A., Bayer, B., Reagan, R. \&. Kallick, B. (2008). El aprendizaje basado en el pensamiento . Madrid: Ediciones SM.

Wales, J. (2015). 'El nuevo analfabetismo no es no saber cosas, es no saber usar la información'. Nota publicada en el diario El Mundo. Recuperado de: http://www.elmundo.es/cultura/2015/10/23/5629fed346163f79308b45c0.html 